\title{
Introducción al Derecho Administrativo alemán
}

\section{Introdution to Germany's Administrative Law}

\author{
COMITÉ EDITORIAL
}

La Universidad Santo Tomás acaba de publicar el libro Introducción al Derecho Administrativo alemán. Esta obra recoge las conferencias que sobre esta temática dictó el profesor Hartmut Maurer, en el seno de la Maestría en Derecho Público que dicha Universidad ofrece en convenio académico con la Universidad de Konstanz, Alemania.

El profesor Maurer es, sin duda, el más destacado y reconocido iuspublicista y Administrativista de Alemania. Su obra científica, particularmente su Tratado de Derecho Administrativo general alemán, ha sido durante décadas -más de dieciocho ediciones- el texto básico para la formación de los juristas de ese país en esa área del Derecho. De esta obra se han hecho traducciones al inglés, francés, portugués, polaco, coreano y chino. Infortunadamente hasta la fecha en lengua castellana no existe ninguna traducción de de los textos jurídicos de tan reconocido tratadista. Por tal motivo, la traducción y publicación de este libro iniciatico al Derecho Administrativo Alemán, que acaba de entregar a la comunidad científica de nuestro país la Universidad Santo Tomás, no sólo constituye un invaluable aporte para el desarrollo de las disciplinas jurídicas, particularmente en el campo del Derecho Administrativo, sino también una verdadera novedad bibliográfica llamada a provocar un importante impacto intelectual en el medio académico colombiano.

Es importante destacar en esta obra la profunda articulación que el profesor Maurer logra entre el Derecho Constitucional, que emana de la Ley Fundamental de Bonn, y el Derecho Administrativo Alemán; entre el Estado Social de Derecho, como fórmula política del estudio alemán, y las instituciones administrativas y su operancia en ese país europeo. Este aspecto debe ser de innegable trascendencia para nuestro ordenamiento jurídico, en el cual, el Derecho Constitucional y el Derecho Administrativo parecen discurrir por vías distintas $y$, en ocasiones enfrentadas, como lo demuestran las constantes colisiones entre la Corte Constitucional y el Consejo de Estado, a propósito de la acción de tutela, así como las divergencias jurisprudenciales entre una y otra corporación en asuntos jurídicos sustanciales. 
Por otra parte, del estudio de los conceptos y de las instituciones administrativas alemanas -fuentes, discrecionalidad de la administración pública, acto administrativo y contrato administrativo-, cabe destacar el análisis del "Principio de protección a la confianza en el Derecho Público". Esta figura, desconocida en nuestro ordenamiento, busca, desde la perspectiva del ciudadano, protegerlo contra cambios intempestivos en las regulaciones estatales y en la jurisprudencia de las Altas Cortes, de suerte que se le compense ante tales eventos, cuando quiera que sus proyectos de vida hayan sido diseñados, confiando en la normatividad existente y en que esta permanecerá estable durante el curso de las actividades proyectadas bajo su imperio.

Mucho se habla y se discute entre nosotros acerca de la seguridad jurídica circunscrita casi exclusivamente a las sentencias de los jueces, sin embargo, se olvida el factor de incertidumbre, que generan los permanentes cambios en la legislación y en las actuaciones administrativas que impiden y dificultan una planificación a mediano y lago plazo de los proyectos -particularmente de tipo económico- de los particulares y sin que tales cambios, que desde luego los afectan, tengan consecuencia alguna para el Estado, piénsese, por ejemplo, en las recurrentes reformas tributarias y su impacto en la marcha de los negocios privados.

Si se tiene en cuenta la metodología comparatista entre los distintos ordenamientos -especialmente el alemán- y el nuestro, el cual orienta la Maestría en Derecho Público de la Universidad Santo Tomás, resultaría altamente provechoso para el país que de ella surgiera una propuesta, a nivel constitucional y legal, que contemplara la introducción en la normatividad nacional del "Principio de protección a la confianza", que amparará al ciudadano contra los cambios abruptos de las regulaciones estatales y garantizará, entre otros efectos posibles, el desarrollo ordenado de las inversiones de los particulares, con miras a alcanzar un desarrollo efectivamente sostenible de nuestra economía.

Éstas, entre muchas, serían las reflexiones que suscita la sintética obra del profesor Maurer sobre el Derecho Administrativo alemán, que con enorme sentido de oportunidad acaba de salir de la imprenta de la Universidad Santo Tomás y que se constituye apenas en un abrebocas para que el lector se pueda introducir en el conocimiento de la numerosa producción intelectual de este reconocido profesor alemán. 


\title{
Los convenios de la administración: entre la gestión pública y la actividad contractual
}

\author{
The agreements of the Administration \\ among Public Management and Contrac- \\ tual Activities
}

En febrero del presente año la Universidad Colegio Mayor de Nuestra Señora del Rosario publicó el libro Los convenios de la administración: entre la gestión pública y la actividad contractual, el cual fue escrito por el profesor de Derecho Administrativo de la Universidad Santo Tomás y de las especializaciones de Derecho Constitucional y de Derecho Administrativo de esa Universidad, Augusto Ramón Chávez. Este texto cuenta con una presentación del reconocido tratadista Libardo Rodríguez Rodríguez.

Por otra parte, esta obra corresponde a la tesis de maestría sustentada como requisito de grado en el curso adelantado en el claustro que ahora la publica, adicionada con unos capítulos que la pusieron al día frente a la nueva Ley 1150 de 2007, mediante la cual se reforma la Ley 80 de 1993, relativa a la contratación estatal; a propósito se debe observar que el contenido de las apreciaciones sobre la figura del convenio van mucho más allá de la visión puramente contractual, como se aprecia en la lectura de las páginas del mencionado libro.

Por el interés científico y académico que representa para el Derecho Administrativo la propuesta del profesor Chávez Marín -autor también del libro Lecturas de Derecho Administrativo, editado por la
Universidad Santo Tomás, cuya segunda edición ya está a disposición del público-, el comité editorial de lusta considera conveniente presentar el texto de la introducción general de Los convenios de la administración: entre la gestión pública y la actividad contractual, a fin de facilitar una visión integral del contenido de la tesis del profesor Chávez Marín, el cual corresponde al siguiente texto:

Un entendimiento progresivo por parte de los administradores de las instituciones públicas sobre la importancia que el acudir a mecanismos asociativos supone para el logro de los fines del Estado, ha hecho en los últimos años de la figura del convenio un instrumento de frecuente utilización en la vida administrativa.

Esa actitud de la administración frente a la necesidad de la utilización de medios asociativos que congreguen el esfuerzo conjunto de diferentes instituciones, se ha desarrollado acudiendo tanto al aporte de entidades del mismo Estado como a organizaciones pertenecientes al sector privado. Por ambos cauces se ha llegado a la configuración progresiva de instrumentos que sirven en la búsqueda de la realización de los intereses de la administración: en el primer caso a los convenios interadministrativos como mecanismos de cooperación, coordinación, apoyo o colaboración entre entidades públicas y, en el segundo, a instrumentos jurídicos que buscan la colaboración de los particulares en contextos que pueden definirse en forma genérica como 
de participación, de administración concertada, de descentralización por colaboración o de fomento de actividades cuya realización interesa tanto al sector privado como al propio Estado.

La referida conducta de la administración pública se sustenta enfáticamente en ciertos principios que tuvieron una especial consagración en la Constitución de 1991. Estos principios marcan el paso hacia un nuevo modelo de Estado en el cual, respecto del interior de la administración, se exige una mayor coordinación y colaboración en el desarrollo de las tareas públicas, y en lo exterior a la misma, se privilegia la democracia participativa y se fomenta, en su contexto, la participación social y ciudadana en la gestión de los intereses generales.

Es así como en el espacio propio del Estado contemporáneo, configurado por la Constitución colombiana de 1991, las mismas administraciones públicas, con miras a la realización de sus objetivos, adoptan un nuevo rol en sus relaciones mutuas, signado por los conceptos de colaboración, cooperación y coordinación en la realización de los intereses que les son comunes, a través de mecanismos de asociación. Entonces adquiere una gran importancia en el desempeño de las tareas públicas la celebración de los tradicionalmente denominados convenios interadministrativos, a los cuales se acude en múltiples campos de una manera cada vez más frecuente, para garantizar la realización de dichos objetivos.

Empero, en el universo de la libertad de empresa, de mercado, de igualdad y de libre competencia que cobija también a las propias administraciones públicas, estas se ubican en el espacio de la oferta de bienes y servicios que pueden ser suministrados por particulares. En tales condiciones, la celebración de un acuerdo de voluntades entre dos instituciones estatales no puede corresponder, muy seguramente, al típico convenio interadministrativo, entendido este como mecanismo apropiado para el logro de intereses mutuos y propios de las instituciones concurrentes; por el contrario, esa figura se ubica mejor, conceptualmente, en el tradicional contrato, que es medio de intercambio patrimonial y plantea intereses contrapuestos entre las partes contratantes, así se trate de dos entidades estatales. Por ello es procedente estudiar si puede distinguirse el convenio interadministrativo (intereses comunes) frente al que cabe identificar como contrato interadministrativo (contradicción de intereses)' ${ }^{1}$.

De otra parte, en el seno de ese nuevo Estado y de su papel en la sociedad de hoy, se hace necesario identificar conceptualmente y contextualizar jurídicamente los mecanismos de asociación de esfuerzos entre el Estado y ciertos sectores de la sociedad civil $^{2}$, asumidos en procura del logro de intereses generales y comunes a las dos instancias y que cobran una mayor vida en el nuevo universo de lo público. Es necesario, para ello, realizar un esfuerzo académico por explorar sus manifestaciones específicas en el ordenamiento actual. A este modo de actuar, a esta figura, a este acuerdo de voluntades celebrado con los particulares, se le puede identificar como convenio administrativo, en tanto es producto de la actividad convencional del Estado y se distingue de la figura de contrato administrativo o estatal, tal cual hoy se le llama, como contraposición de intereses entre las partes contratantes ${ }^{3}$.

A partir de las consideraciones anteriores es procedente formular un planteamiento conforme al cual, en el marco del modelo actual del Estado en Colombia, en el derecho administrativo ha irrumpido en los últimos tiempos una nueva herramienta de gestión pública de naturaleza convencional y no simplemente contractual, que adopta dos modalidades atendiendo a los sujetos que la protagonizan. Ese instrumento jurídico de actuación, que puede identificarse como convenio de la administración, amerita un estudio sistemático y reflexivo sobre su alcance, en orden a construir un universo teórico que lo caracterice

$1 \quad$ El plan de trabajo que se expondrá en el punto siguiente de esta introducción, indicará las partes y capítulos en los que se estudian los conceptos y la tipología de convenios interadministrativos y contratos interadministrativos, y en los que se examinan casos puntuales que evidencian la ocurrencia de dicha comunidad o contradicción de intereses. Vale decir que la ilustración de casos puntuales corresponde al desarrollo de los diferentes tipos de convenios presentados a lo largo de este trabajo. Al llevar a cabo la conceptualización y clasificación que esta tesis propone, se verá cómo en algunos eventos los acuerdos de voluntades celebrados por las personas jurídicas públicas corresponden a verdaderos convenios, mientras que en otros, sustancialmente, se pacta un negocio que puede identificarse como contrato.

2 Para el concepto de sociedad civil, véase parte III, apartado 9.1. de este trabajo.

3 El desarrollo de las ideas que se proponen en este trabajo, permitirá observar los efectos producidos por la distinción que predicamos respecto de las figuras correspondientes al convenio celebrado con particulares (convenio administrativo) y al contrato administrativo o estatal, así como la utilidad que puede derivarse de esa distinción. 
y examine, estableciendo sus especificidades frente al tradicional contrato administrativo.

El convenio ha sido entendido tradicionalmente como una manifestación de la actividad contractual de la administración y se le ha subordinado al contrato, extendiendo al primero el régimen jurídico predicable para el último. Así ocurre con el convenio interadministrativo en el actual Estatuto General de Contratación Estatal contenido en la Ley 80 de 1993. Pero la frecuente utilización del convenio, con fines que no se restringen al intercambio patrimonial propio del contrato administrativo o estatal ${ }^{4}$, así como la complejidad, la dispersión y la ambigüedad de su régimen -que rebasa dicho Estatuto General- justifica para la academia un examen sobre el alcance jurídico de este mecanismo. Por lo tanto, es necesario llevar a cabo una labor de identificación del convenio o de los convenios que celebra la administración, establecer su alcance conceptual y su distinción frente al tradicional contrato administrativo, así como determinar su actual régimen jurídico, lo cual implica asomarse a las figuras de esta especie que el ordenamiento jurídico regula y autoriza ${ }^{5}$.

Con fundamento en la visión anterior, en este trabajo se propone la teoría de los convenios de la administración, bajo la concepción de que esta figura -el convenio de la administración-constituye un género que además de ser un instrumento negocial diferente del contrato administrativo ${ }^{6}$, involucra dos grandes tipos: los celebrados entre las entidades estatales (convenios interadministrativos), y los que se

$4 \quad$ Este aspecto caracterizador del concepto de convenio de la administración se examina en el apartado 2.4. de la parte I de este trabajo, donde se hace una aproximación conceptual a esta categoría general. En el apartado 2.5. se ilumina el tema con el tratamiento del derecho comparado.

5 Desde el punto de vista metodológico, en la anterior exposición se observa cómo esta tesis se propone responder a un problema jurídico central, consistente en establecer si en el marco jurídico actual, constitucional y administrativo, existen unas nuevas herramientas de gestión de naturaleza convencional que ameritan la construcción de una teoría que las examine, caracterice y sistematice. Igualmente, se trata de resolver si el convenio de la administración, como categoría jurídica que engloba dichos instrumentos negociales, constituye una figura con naturaleza y características propias frente al contrato administrativo o estatal, y cuál es su tratamiento jurídico, así como su tipología.

6 La observación sobre la figura del convenio frente a los conceptos de convención y contrato, adoptados por el Código Civil colombiano, y el EGCAP, contenido en la Ley 80 de 1993, se hará en el apartado 2.1. de la parte I. suscriben por ellas con los particulares (convenios administrativos) ${ }^{7}$.

\section{Plan de trabajo}

Para llevar a cabo el estudio de los convenios de la administración este trabajo se dividirá en tres partes. En la parte inicial es necesario primero que todo revisar algunas líneas básicas de la teoría general del contrato administrativo, pues como antes se apuntó el convenio se ha considerado tradicionalmente una manifestación de este negocio jurídico ${ }^{8}$, y hoy se consagra el régimen

$7 \quad$ La relación lógica género-especie entre los conceptos de contrato y convenio, y especialmente la distinción entre convenio de la administración y contrato administrativo, convenio interadministrativo y contrato interadministrativo, así como del convenio administrativo frente al contrato administrativo, se estudian a lo largo de este texto. Sin embargo, véanse al respecto los apartes dedicados especialmente a ello en los apartados 2.1 y 2.4 de la parte I, 4.2 de la parte II y 9.2 de la parte III.

8 SCOGNAMIGLIO, Renato, Teoría general del contrato, Universidad Externado de Colombia, Bogotá, 1996, pp. 13 y 15, explica que la doctrina moderna envuelve dentro del concepto genérico de negocio jurídico todos los actos de autonomía privada relevantes para el derecho, uno de los cuales es el contrato, que corresponde al acto bilateral o plurilateral con contenido patrimonial. Por ello, indica, la figura del contrato representa sólo una de las especies del género negocio, pero constituye la categoría más ampliamente difundida y más representativa del negocio jurídico. En un sentido semejante véase BETTI, Emilio, Teoría general del negocio jurídico, Editorial Revista de Derecho Privado, Madrid, 1959, pp. 51 y 222-228. En Colombia, VALENCIA ZEA, Arturo, Derecho civil, tomo I, Temis, Parte general y personas, Bogotá, 1976, pp. 500-525, basado en la doctrina alemana, clasifica los hechos jurídicos en voluntarios e involuntarios; a los primeros los denomina actos jurídicos; los actos jurídicos, a su vez, pueden ser ilícitos o ilícitos; los actos jurídicos lícitos se subdividen en actos derechos y en declaraciones de voluntad a las que llama también negocios jurídicos. Señala que los negocios jurídicos son unilaterales o plurilaterales. En estos últimos ubica el contrato, remitiéndose al artículo 1495 del Código Civil y después de haber recordado que la doctrina colombiana emplea la expresión negocio jurídico para referirse a los hechos jurídicos consistentes en las declaraciones de voluntad. Por lo tanto, para Valencia Zea también el contrato es un negocio jurídico, como se evidencia en el curso de su exposición; por lo demás, el mismo autor recuerda que "En las doctrinas alemana, francesa y suiza, el contrato se identifica con el término de negocio bilateral; son contratos, tanto los negocios obligatorios como los dispositivos".

De otra parte, el concepto de negocio jurídico puede ser trasladado al derecho administrativo y utilizado para referirse a los contratos administrativos o estatales, si además de lo anterior se atiende la esencia de esa figura consistente en la concurrencia de declaraciones de voluntad que por sí o unida a otros requisitos, persigue determinados efectos jurídicos, como lo señala Valencia Zea, op. cit., p. 504. Ello si se atiende que conforme al artículo 32 del EGCAP, los contratos administrativos o estatales son todos los actos jurídicos generadores de obligaciones que celebren las entidades estatales, previstos en el derecho privado o en disposiciones especiales, o derivados del ejercicio de la autonomía de la voluntad. 
general del mismo en el EGCAP, contenido en la Ley 80 de 1993 y en la Ley 1150 de 2007 (capítulo 1). Hecho lo anterior, se hará una aproximación a la figura del convenio de la administración como categoría general y mecanismo de gestión pública del que puede predicarse un importante grado de distinción y autonomía respecto del contrato administrativo o estatal. En virtud de ello se delimitará el concepto de convenio de la administración, precisando sus rasgos distintivos y característicos (capítulo 2).

La segunda parte de esta obra estará dedicada al examen de la categoría de los convenios interadministrativos y algunas de sus más importantes modalidades. Allí se observará cómo esa figura se plantea en el marco de las relaciones externas o intersubjetivas de la administración y se distingue de los que en este trabajo se denominan acuerdos interorgánicos, denominación que se explica en el capítulo $3^{9}$. Estos últimos se presentan en el campo de las relaciones internas o interorgánicas; por lo cual se hace necesario estudiar la teoría del órgano, la cual sirve para identificar las dos clases de relaciones enunciadas (capítulo 3).

Una vez hecha una aproximación conceptual a la figura del convenio interadministrativo $y$ analizada su distinción con el contrato interadmi-

Como en esta obra se hace un esfuerzo de clasificación y tipificación de los diferentes convenios que celebra la administración, el autor propondrá, tanto para las categorías genéricas como para las especies, la asignación de denominaciones que los distingan e identifiquen frente a las demás figuras del mundo jurídico. En efecto, como se verá a lo largo de este trabajo, en la medida en que se vayan identificando y distinguiendo las distintas expresiones de la actividad convencional, se propondrá una nominación de figuras jurídicas como ocurre con el convenio de la administración, convenio administrativo, acuerdo interorgánico, convenio público de organización, convenio de delegación, convenio interadministrativo de colaboración. Los nombres que se vayan asignando, tendrán, por supuesto, la justificación correspondiente en cada oportunidad. En las conclusiones de cada una de las tres partes en que se divide este trabajo, así como en las conclusiones generales, se recapitulará la clasificación que resulte de esas identificaciones. Ese esfuerzo de nominación es necesario en cuanto en la mayoría de los casos la ley y la doctrina, cuando se han ocupado del tema, guardan silencio sobre el nombre de tales figuras. Esta es, por supuesto, una tarea que corresponde a la dogmática jurídica y cuya responsabilidad no puede eludirse, en el ánimo de sistematizar el conocimiento de este universo. "Sin vocabulario jurídico no hay derecho", la identidad del lenguaje es un instrumento primordial en la tarea de la doctrina y la jurisprudencia, nos recuerda RIVERO, Jean, Páginas de derecho administrativo, Temis-Universidad del Rosario, Bogotá, 2002, p. 58. nistrativo (capítulo 4), se examinará la regulación general o común de los convenios interadministrativos y los contratos interadministrativos, contenida básicamente en el EGCAP (capítulo 5). Para tal fin y dado que en la Ley 1150 de 2007, por la cual se reforma la Ley 80 de 1993, se introducen una serie de normas generales sobre los que allí se identifican como contratos interadministrativos, es necesario hacer un examen sobre su alcance específico. A su vez, para llevar a cabo tal estudio es preciso hacer una referencia, así sea de orden general, a las principales previsiones de la citada reforma, a efecto de ubicar al lector en el marco normativo en el cual se presentan las nuevas normas sobre esta materia.

Realizado así el examen del régimen común de los contratos y convenios interadministrativos en dicho capítulo 5, en el siguiente se llevará a cabo un estudio de dos interesantes casos que han tenido una importancia singular en el derecho colombiano, que ilustran bien la discusión planteada en este trabajo y sobre los cuales la Ley 1150 de 2007 ha concentrado especialmente su atención. Ellos son los contratos y convenios celebrados con entidades estatales cooperativas y los suscritos por las entidades estatales con organismos internacionales de cooperación, asistencia o ayuda (capítulo 6).

En seguida, se llevará a cabo un acercamiento a una propuesta de tipología de los convenios interadministrativos. En este capítulo se revisarán los convenios interadministrativos de cooperación, los convenios de asociación, los convenios públicos de organización y los convenios interadministrativos de desempeño (capítulo 7). Al final de la segunda parte se centrará la atención en uno de los tipos más relevantes de esta categoría, cual es el convenio de delegación (capítulo 8).

Sobre dos puntos debe llamarse la atención en relación con esta segunda parte del texto: el primero tiene que ver con la tipología de los convenios interadministrativos, respecto de lo cual es interesante señalar que la examinada en este trabajo se concentra en la novedosa Ley 489 de 1998, lo cual no significa que no existan otros tipos de convenios interadministrativos en otras áreas del derecho administrativo. Así por ejemplo, son bien conocidos en la administración pública los denominados convenios de cofinanciación, acuerdos que constituyen verdaderos 
convenios interadministrativos y tienen una gran relevancia dentro del ámbito jurídico colombiano por el papel que desempeñan en el desarrollo territorial $^{10}$. Sin embargo, puede afirmarse que es en la Ley 489 de 1998 en la cual el convenio de la administración, como categoría general, así como en sus dos modalidades básicas, obtiene pleno reconocimiento.

En relación con el alcance de este trabajo, resulta conveniente precisar que el mismo recoge una tesis expuesta para optar por el título otorgado en un curso de maestría en derecho administrativo. Parece propio de una tesis del nivel académico superior, abrigar y llevar a cabo el propósito de agotar en todos sus matices y manifestaciones el tema que se aborda como objeto de investigación (si ello es posible aún en esos eventos). Siendo claro que el presente trabajo, como se dijo, corresponde a una tesis de maestría, en la cual se hace una propuesta de solución frente a un problema que se ha detectado en los ámbitos de la legislación, la jurisprudencia, la doctrina y la práctica jurídico-administrativa, ante la multiplicidad de manifestaciones tipológicas que presentan las figuras que aquí se exploran, el autor considera pertinente proponer los conceptos que sustentan las categorías básicas referidas al convenio interadministrativo y el convenio administrativo; y con el fin de demostrar la validez que en el derecho positivo tienen las mismas y de hacer su muestreo tipológico, estima igualmente necesario abordar el examen específico de algunos de los tipos que ilustran la existencia de esos modelos teóricos. Todo ello, en consecuencia, no significa de ninguna manera desconocer la importancia que pueden tener las múltiples manifestaciones convencionales que se dejan de lado por las razones indicadas.

En segundo lugar, en la segunda parte del texto será pertinente distinguir los convenios interadministrativos de aquellos compromisos que se identifican como contratos interadministrativos, lo cual se hará con fundamento en la teoría de la causa y a partir de una perspectiva que supere la visión meramente formal de la actividad contractual de la administración; todo ello atado y basado en la propia delimitación del concepto

10 Dada la complejidad y la importancia de este tipo de compromisos, su examen merece una atención especial y monográfica que no se abordará en esta investigación. de convenio de la administración. A partir de la consideración anterior se asigna a cada una de estas dos figuras -convenios interadministrativos y contratos interadministrativos- un alcance distinto, y se propone la necesidad de replantear el tratamiento unánime que los dos tipos de actividad reciben en el actual estatuto contractual, en cuanto ellos corresponden a instrumentos sustancialmente diferentes aunque formalmente semejantes. En el marco de la exposición de la segunda parte se señala la frecuente existencia en la práctica administrativa de contratos interadministrativos, práctica en la que subyace una concepción que requiere ser precisada, conforme a la cual esta figura constituye una modalidad contractual distinta del convenio interadministrativo y debiera tener un tratamiento legal diferente de éste último instrumento de gestión administrativa ${ }^{11}$.

La tercera parte de esta tesis se centra en el estudio de la segunda categoría general de los convenios de la administración: la de los convenios administrativos. En el capítulo 9 se presentarán los fundamentos conceptuales y constitucionales de la figura, para luego hacer una aproximación conceptual a ese mecanismo, estableciendo algunos de sus rasgos específicos con el ánimo de identificar su alcance y delimitación jurídica.

La revisión del derecho administrativo colombiano permite señalar que a la categoría de los convenios administrativos pertenecen una serie de tipos de negocios jurídicos, entre los cuales se destacan los convenios administrativos de colaboración, regulados por el capítulo 16 de la Ley 489 de 1998 (capítulo 10), y los convenios administrativos de interés público, autorizados por el inciso 2 del artículo 355 de la Constitución y regulados por normas especiales dictadas por

11 Las ideas que se plantean en la segunda parte del trabajo, oscilan entre la descripción y la prescripción, entre el examen de lo que es nuestro derecho positivo y entre lo que debe ser. Allí se propone una visión distinta de las cosas para regular la materia de una mejor manera y de una forma más conveniente a los intereses de la sociedad. Es conocida entre los servidores públicos la utilización frecuente de la figura del convenio interadministrativo, para encubrir bajo su manto la celebración de verdaderos contratos administrativos y eludir así la aplicación de todo el régimen contractual, especialmente el de selección de los contratistas de la administración. La aplicación de este régimen sería claramente exigible en el evento de que se tratara abiertamente de un contrato administrativo bajo la modalidad de contrato interadministrativo, como en esta obra se propone. 
el gobierno nacional en desarrollo de esa misma disposición (capítulo 11) ${ }^{12}$.

Finalmente, en la tercera parte de este trabajo se avanzará sobre un régimen en relación con el cual no se conocen estudios en la doctrina colombiana, no obstante que en la práctica administrativa es frecuente su utilización y toma cada vez una mayor importancia por el avance de este campo en la sociedad actual marcada por la informática: el de las actividades de ciencia y tecnología. En efecto, esa normatividad especial integrada por la Ley 29 de 1990 y los Decretos extraordinarios 393 y 591 de 1991, regula una serie de figuras que tienen relación con las estudiadas en este trabajo. En estas normas, ricas y variadas en mecanismos contractuales y convencionales, se pueden identificar casos como los del convenio especial de cooperación en ciencia y tecnología, el convenio de financiamiento de ciencia y tecnología, los convenios de organización en ciencia y tecnología, así como una figura propiamente contractual que es el contrato de administración de proyectos en ciencia y tecnología. A todos ellos se dedicará el capítulo 12, último de la tercera parte.

El esfuerzo de clasificación e identificación tipológica abordado en las partes segunda y tercera, se presentará conceptualmente en las conclusiones de cada una de esas partes y esquemáticamente en las conclusiones generales de la obra.

\section{Justificación del presente trabajo}

El derecho administrativo ha evolucionado desde su nacimiento bajo diferentes modelos de Estado, y ha dado curso a través de su regulación a distintas situaciones en las cuales ha desempeñado la función social propia de un conjunto normativo. En el curso de esa evolución, asímismo,

12 La explicación de la denominación de este último tipo de convenios administrativos se encontrará en el capítulo que se acaba de indicar; no obstante, parece oportuno adelantar que obedece a la identificación hecha por la norma constitucional citada, en el sentido de que deben celebrarse para el desarrollo de programas y actividades de interés público. La utilización de esta expresión para identificar esa clase de compromisos no debe entenderse en el sentido de que los demás convenios de la administración no tengan por objeto actividades que conlleven también el interés público, sólo que en el caso de dichos acuerdos tal carácter tiene un énfasis distintivo frente a las actividades de interés privado que ordinariamente realizan los particulares. se ha perfilado como un área específica de este derecho la referente a la teoría del contrato administrativo, y ha delimitado progresivamente su identidad frente a la del contrato celebrado entre los particulares y a la regulación del derecho privado. En nuestro medio, a su vez, al interior de la teoría del contrato administrativo, se ha considerado como una de las manifestaciones de este el denominado convenio interadministrativo, y así lo ha recogido el derecho positivo.

Sin embargo, como antes se indicó, en los últimos años el tratamiento del convenio por parte del derecho positivo ha desbordado el marco propio del contrato administrativo, y ha adquirido ribetes específicos que sugieren la existencia de una nueva manifestación de la actividad administrativa. Este hecho, sucedido con mayor énfasis en la década de los años noventa, impulsado por el nuevo modelo de Estado que propuso la Constitución de 1991, así como por sus normas específicas en ciertas materias y por sus desarrollos legislativos o reglamentarios, como los contenidos en la Ley 489 de 1998 o en los reglamentos constitucionales dictados por el gobierno nacional en desarrollo del artículo 355 de la Carta, implica una revaloración de la actividad contractual de la administración y la necesidad de explorar la existencia de la actividad convencional protagonizada por ella. En este contexto, no puede desconocerse que la expresión de la gestión administrativa a través del convenio se ha visto sustentada por una práctica reiterada de la utilización de esta figura con unos fines que superan el puro interés patrimonial o contraprestacional atribuido tradicionalmente al contrato administrativo.

La exploración y el análisis de estos problemas que parten de la teoría del contrato administrativo y se adentran en el examen específico del convenio como manifestación autónoma de la gestión administrativa, observados en el contexto de una nueva visión constitucional de lo público y lo privado y en el marco de un nuevo modelo de Estado, justifican una investigación como la que en este documento se propone. Debe consistir en una indagación que, entre otras cosas, realice un esfuerzo de certidumbre frente a esta nueva categoría cuyo estudio sistemático hace falta en la doctrina nacional.

De acuerdo con lo anterior, en este trabajo se estima necesario adelantar un examen de exploración conceptual tendiente a establecer 
desde la dogmática del derecho administrativo la prosperidad de la tesis de que los convenios de la administración constituyen una categoría distinta del contrato administrativo o estatal, al interior de la cual se presenta toda una tipología que merece estudio e identificación. Tal es el esfuerzo académico al que se dedica el autor, y su necesidad parece estar justificada por las razones que preceden.

Adicionalmente, la concepción del convenio y del contrato que se expone en este documento permite una aproximación a la idea de la necesidad de regular ciertas especies de negocios jurídicos de forma distinta a la que hoy presenta el derecho positivo. Se piensa que modificar aspectos de ese régimen puede hacer más acorde tales figuras con principios de transparencia y pulcritud, reconducir la administración por caminos de eficacia y economía en el manejo de los intereses públicos, así como propiciar una mejor satisfacción de las necesidades administrativas y sociales, y una mayor concordancia con los postulados de la Constitución de 1991.

\section{Antecedentes de esta teoría}

Sorprende el hecho de que, salvo muy contados autores, la doctrina colombiana haya sido parca en el estudio monográfico de este nuevo tema que el derecho administrativo enfrenta ${ }^{13}$. Es así

13 Véase el artículo del autor de este trabajo "Los convenios de la administración" en El Ágora, №2, Bogotá, enero/junio de 1998, incluido también en el libro Lecturas de derecho administrativo, Universidad Santo Tomás, Bogotá, 2005; y PINNO RICCl, Jorge, "Los convenios interadministrativos" en Jornadas internacionales de derecho administrativo, Universidad Externado de Colombia, Bogotá, 2001. Este último es uno de los múltiples artículos incorporados en el libro que reúne las ponencias presentadas en dichas jornadas, pero referido especialmente a los convenios interadministrativos, recoge y desarrolla el esquema que planteamos en 1998 en la revista mencionada, ilustrando sus alcances con los autores extranjeros que allí citamos. Es interesante observar cómo en Colombia este tema no ha sido estudiado con el detenimiento que ha merecido en otras latitudes. Los autores de textos generales sobre contratación estatal se refieren al convenio interadministrativo solamente al identificarlo para efectos del régimen especial que en algunos aspectos le asigna el EGCAP, pero no se profundiza en el alcance de esa figura. El mayor acercamiento a su concepto, pero con visión comparatista y con el interés puntual que inspira esa investigación, se puede encontrar en EXPÓSITO VÉLEZ, Juan Carlos, La configuración del contrato de la administración pública en derecho colombiano y español, Universidad Externado de Colombia, Bogotá, 2003. como la revisión de la materia es presentada, especialmente en sus rasgos básicos, por los textos generales del derecho de la contratación administrativa. La jurisprudencia del Consejo de Estado y de la Corte Constitucional, igualmente, en contadas ocasiones ha revisado la materia $y$, por lo demás, lo han hecho de una manera tangencial. Solamente con ocasión de demandas contra normas de la Ley 489 de 1998, la Corte tuvo la oportunidad de pronunciarse en relación con ciertos aspectos puntuales previstos por esa ley respecto de algunos convenios, pero sin profundizar en el alcance de la figura y su régimen específico.

La escasez doctrinaria y jurisprudencial que acabamos de mencionar, se presenta como una insuficiencia que se llena ordinariamente acudiendo a las obras generales mencionadas $y$, especialmente, a la doctrina extranjera, la cual ha sido más abundante en esta materia. Por ejemplo, sobre los convenios celebrados entre administraciones públicas se destaca en la doctrina foránea la tesis doctoral de José María Rodríguez Santiago, Los convenios entre administraciones públicas (Marcial Pons, Madrid, 1997), mientras en relación con los convenios celebrados por las entidades estatales con los particulares se encuentra la obra Los convenios urbanísticos, escrita por Alejandro Huergo Lora (Civitas, Madrid, 1998), autor del cual también puede consultarse el trabajo de doctorado Los contratos sobre los actos y potestades públicas (Civitas, Madrid,1998).

Por lo anterior, al adelantar un trabajo monográfico como el aquí propuesto, es a veces necesaria la remisión a la literatura extranjera, lo cual no implica, en modo alguno, que se pretenda adelantar un estudio de derecho comparado, sino que se aborda la manera como ciertos aspectos de la materia han sido regulados por el derecho foráneo o concebidos por los publicistas de otros países.

De otra parte, es muy familiar al lenguaje jurídico colombiano, en el área del derecho administrativo, la expresión convenios interadministrativos, y su existencia se entiende claramente reconocida por la ley, la doctrina y la práctica administrativa. Pero también la figura del convenio se utiliza permanentemente en el sector privado como un mecanismo de alianza estratégica. Asímismo, en los últimos años ha tomado fuerza su uso para acordar asuntos entre el sector público y 
la sociedad civil o el sector privado. Al acudir a esta figura, tanto en el sector público como en el privado, se le concibe como un mecanismo que tiene rasgos distintos a la figura tradicional del contrato y que merece ser denominado de esta forma, es decir, como convenio.

Este trabajo, ubicado en el campo del derecho administrativo, pretende recoger ese tópico, esto es, esa significación generalmente aceptada por la sociedad, que se le viene asignando a la figura del convenio ${ }^{14}$. A partir de ese entendimiento se construye la teoría que en esta obra se propone. Parece válido considerar que esa teoría ha sido acogida por nuestra legislación, en cuanto el ordenamiento jurídico ha regulado distintos tipos de convenios, tanto de aquellos celebrados entre personas jurídicas públicas (interadministrativos), como de los que se suscriben entre el Estado y los particulares (convenios administrativos).

\section{Algunas observaciones sobre el método}

Dos observaciones de orden metodológico conviene hacer en esta introducción. La primera, que ya se avizora en las anteriores observaciones, se refiere al carácter deductivo del trabajo adelantado. Ante todo es necesario aclarar que este enfoque metodológico obedeció a una opción del autor, pues si bien es el que se consideró más apropiado para el desarrollo del tema, era posible optar por una mirada inductiva del objeto estudiado. Sin embargo, aunque este último camino hubiera sido posible, no era el más aconsejable y probablemente el resultado de la investigación no sería tan legible como el autor espera que sea el logrado ${ }^{15}$.

El método deductivo ha permitido, en la primera parte del trabajo, llevar a cabo la identificación del concepto y las características del convenio de la administración, el cual constituye una categoría general. Teniendo como sustento ese concepto, se desciende en la primera y en la segunda parte al estudio de cada una de las dos grandes especies en las que se divide ese género, las cuales, como se ha indicado, corresponden al convenio interadministrativo y al convenio administrativo. En tales partes se presentan no solamente las especificidades de las dos especies, sino que se hace una aplicación de las características y avances logrados en la identificación del género examinado en la primera parte.

Más aún, al interior de las partes segunda y tercera, una vez establecida la identificación del convenio interadministrativo y del administrativo, se baja en la escala conceptual hacia una aplicación específica en el derecho positivo de cada una de esas categorías. En estos casos se puede observar que las clases de convenio interadministrativo y de convenio administrativo, gozan a su vez no solo de las características del género convenio de la administración, sino de la modalidad a la que pertenecen y concretan en el universo jurídico cada una de las subespecies correspondientes.

14 Sobre el concepto de topoi o tópico al que acudimos, se puede consultar VIEHWEG, Theodor, Tópica y jurisprudencia, Taurus, Madrid, 1964, p. 38; GARCÍA AMADO, Juan Antonio, Tópica y jurisprudencia, Civitas, Madrid, 1988, p. 364; y CHÁVEZ MARÍN, Augusto Ramón, Dos estudios de tópica jurídica, Procuraduría General de la Nación, Bogotá, 2004, p. 19.

15 En efecto, era posible un estudio inductivo del tema: ascender de lo particular a lo general, esto es, a partir de ciertas manifestaciones específicas de convenios interadministrativos y convenios administrativos existentes en el ordenamiento jurídico, inducir la existencia de estas dos figuras y a partir de ellas la de la categoría general que es el convenio de la administración. 